\title{
EIGENVALUES OF MATRICES WITH PRESCRIBED ENTRIES
}

\author{
DAVID LONDON AND HENRYK MINC ${ }^{1}$
}

\begin{abstract}
It is shown that there exists an $n$-square matrix all whose eigenvalues and $n-1$ of whose entries are arbitrarily prescribed. This result generalizes a theorem of L. Mirsky. It is also shown that there exists an $n$-square matrix with some of its entries prescribed and with simple eigenvalues, provided that $n$ of the nonprescribed entries lie on a diagonal or, alternatively, provided that the number of prescribed entries does not exceed $2 n-2$.
\end{abstract}

A well-known result of L. Mirsky [3] states essentially that, given any $2 n-1$ complex numbers $\lambda_{1}, \cdots, \lambda_{n}, a_{1}, \cdots, a_{n-1}$, there exists an $n$ square matrix with eigenvalues $\lambda_{1}, \cdots, \lambda_{n}$ and $n-1$ of its main diagonal entries equal to $a_{1}, \cdots, a_{n-1}$. Related results for matrices over general fields were also obtained by Farahat and Ledermann [1].

We show that the restriction of the $n-1$ prescribed entries to the main diagonal is unnecessary. We first investigate the conditions under which there exists a matrix with prescribed entries and simple eigenvalues.

A position in a matrix in which some entries have been prescribed is said to be free, if there is no prescribed entry in that position. By a diagonal in an $n$-square matrix we mean a set of $n$ positions no two of which are in the same row or in the same column; i.e., positions $(i, \sigma(i))$, $i=1, \cdots, n$, for some permutation $\sigma$.

THEOREM 1. Let $a_{1}, \cdots, a_{n^{2}-n}$ be $n^{2}-n$ prescribed complex numbers and let $\left(i_{t}, j_{t}\right), t=1, \cdots, n^{2}-n$, be prescribed different positions in an $n$-square matrix, such that the $n$ remaining free positions form a diagonal of the matrix. Then there exists an $n$-square matrix with simple eigenvalues and with the prescribed entries $a_{t}$ in the prescribed positions $\left(i_{t}, j_{t}\right), t=1, \cdots$, $n^{2}-n$. The number $n$ of free positions cannot, in general, be decreased.

Received by the editors June 1, 1971.

AMS 1969 subject classifications. Primary 1525.

Key words and phrases. Eigenvalues, matrices, prescribed entries, prescribed eigenvalues, simple eigenvalues.

${ }^{1}$ The research of this author was supported in part by the U.S. Air Force Office of Scientific Research under Grant AFOSR 72-2164.

(c) American Mathematical Society 1972 
Proof. Let the free positions be $(i, \sigma(i)), i=1, \cdots, n$, where $\sigma$ is a permutation of $(1, \cdots, n)$. Let

$$
\sigma=C_{1} C_{2} \cdots C_{r}
$$

be the representation of $\sigma$ as the product of the disjoint cycles $C_{k}$, and let $n_{k}, k=1, \cdots, r$, be the length of $C_{k}$.

A permutation on the rows of a matrix followed by the same permutation on the columns leaves the eigenvalues unchanged. Hence, applying a suitable permutation, we may assume without loss of generality that the cycles $C_{k}$ are

$$
\begin{aligned}
& C_{1}=\left(1, \cdots, n_{1}\right), \\
& C_{2}=\left(n_{1}+1, \cdots, n_{1}+n_{2}\right), \\
& \dot{\cdot} \\
& \dot{C_{r}}=\left(n_{1}+\cdots+n_{r-1}+1, \cdots, n\right) .
\end{aligned}
$$

That is, we assume that the free positions are $(i, \sigma(i)), i=1, \cdots, n$, where $\sigma$ is given by (1), and the $C_{k}$ are defined in (2).

Denote by $P_{k}, k=1, \cdots, r$, the $n_{k}$-square full cycle permutation matrix:

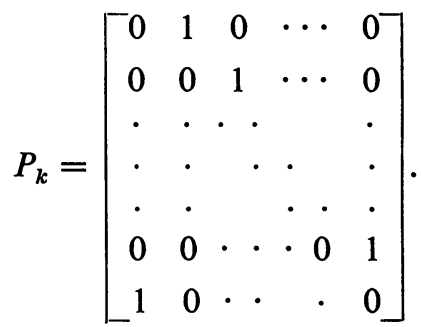

Let $B=\left(b_{i j}\right)$ be the $n$-square matrix defined by the direct sum

$$
B=P_{1}+2 P_{2}+\cdots+r P_{r} \text {. }
$$

The eigenvalues of $k P_{k}, k=1, \cdots, r$, are $\lambda_{h}^{(k)}=k \exp \left(2 \pi i h / n_{k}\right), h=1, \cdots$, $n_{k}$. Hence, all the eigenvalues of $B$ are simple.

Let $\alpha$ be a real number and define the $n$-square matrix $A(\alpha)=\left(a_{i j}(\alpha)\right)$ by

$$
\begin{aligned}
a_{i j}(\alpha) & =\alpha b_{i j}, & & \text { if } j=\sigma(i), \\
& =a_{t}, & & \text { if } i=i_{t}, j=j_{t}, t=1, \cdots, n^{2}-n .
\end{aligned}
$$

Note that $A(\alpha)$ has the prescribed entries in the prescribed positions. It is obvious that

$$
\lim _{\alpha \rightarrow \infty}(A(\alpha) / \alpha)=B
$$


As all the eigenvalues of $B$ are simple, it follows from (4) that, for a sufficiently large $\alpha$, all the eigenvalues of $A(\alpha) / \alpha$, and hence of $A(\alpha)$, are simple.

Thus for such an $\alpha$ the matrix $A(\alpha)$ has the appropriate prescribed entries and simple eigenvalues. We complete the proof by showing that the number of free entries cannot, in general, be decreased, even in case they belong to the same diagonal. For example, if $a_{t}=0, t=1, \cdots$, $n^{2}-n+1$, and if the remaining $n-1$ free positions are $(i, i+1), i=1, \cdots$, $n-1$, then regardless of the choice of the free entries, the eigenvalues of the matrix are all 0 .

COROllary. Let $x_{i}, i=1, \cdots, n$, be given complex numbers, and let the prescribed entries and positions be as in Theorem 1. Then there exists an $n$-square matrix $A=\left(a_{i j}\right)$ with simple eigenvalues, such that $A$ has the prescribed entries in the prescribed positions, and such that the free entries $a_{i \sigma(i)}$ are arbitrarily close to $x_{i}, i=1, \cdots, n$.

Proof. We regard $A$ as a function of its free entries $a_{i \sigma(i)}, i=1, \cdots, n$, and we consider the discriminant $\Delta$ of the characteristic polynomial of $A$. $\Delta=\Delta\left(a_{1 \sigma(1)}, \cdots, a_{n \sigma(n)}\right)$ is a polynomial in $a_{i \sigma(i)}$, and it vanishes if and only if $A$ has a multiple eigenvalue. If the corollary were not true, then $\Delta$ would vanish identically in some neighborhood of the point $\left(x_{1}, \cdots, x_{n}\right)$, and thus would vanish in the whole space. Hence, $A$ would have multiple eigenvalues for all choices of the free entries. But this would contradict Theorem 1.

THEOREM 2. Let $a_{1}, \cdots, a_{2 n-2}$ be $2 n-2$ prescribed complex numbers and let $\left(i_{t}, j_{t}\right), t=1, \cdots, 2 n-2$, be prescribed different positions in an $n$-square matrix. Then there exists an $n$-square matrix with simple eigenvalues and with the prescribed entries $a_{t}$ in the positions $\left(i_{t}, j_{t}\right), t=1, \cdots$, $2 n-2$. The constant $2 n-2$ cannot in general be increased.

Proof. If there exists a diagonal all of whose entries are free, then our assertion follows from Theorem 1 .

If such a diagonal does not exist, it follows from the Frobenius-König theorem (see, e.g., [2]) that there exists an $s \times t$ submatrix consisting entirely of prescribed positions such that $s+t=n+1$.

Since $s t \leqq 2 n-2$, only the following two cases need to be considered:

(i) $s=1, t=n$;

(ii) $s=2, t=n-1$.

Case (i). There exists a complete row consisting of prescribed positions; we may assume that it is the last row. Consider the prescribed positions $\left(i_{t}, j_{t}\right)$ for which $1 \leqq i_{t} \leqq n-1$ and $1 \leqq j_{t} \leqq n-1$. There are at most $n-2$ such positions. Hence, there exists a permutation $\tau$ of $(1, \cdots, n-1)$ 
for which the positions $(i, \tau(i)), i=1, \cdots, n-1$, are free. Thus, as in the proof of Theorem 1, we can construct an $(n-1)$-square matrix $A^{\prime}(\alpha)=$ $\left(a_{i j}^{\prime}(\alpha)\right)$ such that all the prescribed entries are in the prescribed positions $\left(i_{t}, j_{t}\right), 1 \leqq i_{t}, j_{t} \leqq n-1$, and the entries in the free positions outside the diagonal $(i, \tau(i)), i=1, \cdots, n-1$, are chosen to be 0 . We have

$$
\lim _{\alpha \rightarrow \infty}\left(A^{\prime}(\alpha) / \alpha\right)=B^{\prime}
$$

where $B^{\prime}$ has simple eigenvalues different from 0 . Define

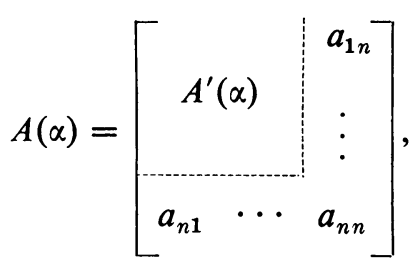

where all the entries in the last row, and possibly certain entries in the last column, are prescribed. The other (free) entries in the last column are chosen to be 0 . We have,

$$
\lim _{\alpha \rightarrow \infty} \frac{A(\alpha)}{\alpha}=\left[\begin{array}{cc:c}
B^{\prime} & \vdots \\
& & \vdots \\
\hdashline 0 & \cdots & 0
\end{array}\right] .
$$

As all the eigenvalues of the limit matrix are simple, the same is true for $A(\alpha)$, for $\alpha$ large enough, and the proof of Case (i) is completed.

Case (ii). All the prescribed positions lie in an $2 \times(n-1)$ submatrix. We may assume that this submatrix belongs to the first two rows.

If the free positions in the first two rows are either $(1,1)$ and $(2,1)$, or $(1,2)$ and $(2,2)$, then the free elements can be chosen in an obvious way to give a matrix with simple eigenvalues.

Now suppose that the free positions in the first two rows are $(1, k)$ and $(2, k)$ for some $k, 3 \leqq k \leqq n$. Define the $n$-square matrix $A(\alpha)=\left(a_{i j}(\alpha)\right)$ by:

$$
\begin{aligned}
& a_{t t}(\alpha)=t \alpha, \quad t=3, \cdots, k-1, k+1, \cdots, n, \\
& a_{1 k}(\alpha)=a_{k 1}(\alpha)=\alpha, \\
& a_{2 k}(\alpha)=0
\end{aligned}
$$

all the other entries in the first two rows have the prescribed values, and all the entries not specified above are equal to 0 . Then the matrix $\lim _{\alpha \rightarrow \infty}(A(\alpha) / \alpha)$ has entries equal to $t$ in the $(t, t)$ positions, $t \neq 1, t \neq 2$, $t \neq k$; it has 1 's in the $(1, k)$ and the $(k, 1)$ positions, and zeros elsewhere. 
Hence its eigenvalues are simple; in fact, they are: $0,-1,1,3,4, \cdots$, $k-1, k+1, \cdots, n$. The conclusion follows as in Case (i).

Finally, we show that the number $2 n-2$ cannot in general be increased. Let $a_{t}=0, t=1, \cdots, 2 n-1$, and let the $2 n-1$ prescribed positions be $(1, j), j=1, \cdots, n$, and $(2, j), j=2, \cdots, n$. Then for all choices of the free entries, the resulting matrix has a multiple root 0 .

TheOREM 3. Let $\lambda_{1}, \cdots, \lambda_{n}$ and $a_{1}, \cdots, a_{n-1}$ be prescribed complex numbers and let $\left(i_{t}, j_{t}\right), t=1, \cdots, n-1$, be prescribed different positions in an $n$-square matrix. Then there exists an $n$-square matrix with eigenvalues $\lambda_{1}, \cdots, \lambda_{n}$ and with the prescribed entries $a_{t}$ in the prescribed positions $\left(i_{t}, j_{t}\right), t=1, \cdots, n-1$. The number $n-1$ of prescribed entries cannot in general be increased.

Proof. Use induction on $n$. If $n=2$ then the result clearly holds. For, if the prescribed entry is not on the main diagonal, then we can choose a triangular matrix with the prescribed eigenvalues, and if it is on the main diagonal, e.g., in the $(1,1)$ position, then the matrix

$$
\left[\begin{array}{ll}
a_{1} & c \\
1 & b
\end{array}\right]
$$

where $b=\lambda_{1}+\lambda_{2}-a_{1}$ and $c=a_{1} b-\lambda_{1} \lambda_{2}$, has eigenvalues $\lambda_{1}$ and $\lambda_{2}$.

We assume now that the theorem holds for $(n-1)$-square matrices and prove the result for $n$-square matrices with $n-1$ prescribed entries. Since there are only $n-1$ pairs of integers $\left(i_{1}, j_{1}\right), \cdots,\left(i_{n-1}, j_{n-1}\right)$, not every integer $1,2, \cdots, n$ can appear more than once among the numbers $i_{1}, j_{1}, i_{2}, j_{2}, \cdots, i_{n-1}, j_{n-1}$. Hence, there must exist an integer $s, 1 \leqq s \leqq n$, that occurs at most once; that is to say, an integer $s$ such that either (i) there is exactly one prescribed entry in the row $s$ or in the column $s$, but not both, or (ii) there are no prescribed entries in row $s$ and column $s$. We can assume without loss of generality that $s=n$.

Case (i). There is exactly one prescribed entry in the last column and no prescribed entry in the last row, or vice versa. We can assume without loss of generality that $i_{n-1}=n$ and $j_{n-1}=k, 1 \leqq k \leqq n-1$. By the induction hypothesis, there exists an $(n-1)$-square matrix $A^{\prime}=\left(a_{i j}^{\prime}\right)$ with eigenvalues $\lambda_{1}, \cdots, \lambda_{n-1}$ and $n-2$ prescribed entries $a_{i_{t} j_{t}}^{\prime}=a_{t}, t=1, \cdots, n-2$. The matrix

$$
A=\left(a_{i j}\right)=\left[\begin{array}{c:c} 
& 0 \\
A^{\prime} & \vdots \\
& 0 \\
\hdashline 0 \cdots 0 a_{n-1} 0 \cdots 0 & \lambda_{n}
\end{array}\right]
$$


in which $a_{n k}=a_{n-1}, a_{n n}=\lambda_{n}$ and all the other entries in the last row and in the last column are 0 , has eigenvalues $\lambda_{1}, \cdots, \lambda_{n-1}, \lambda_{n}$ and all the prescribed entries in the prescribed positions.

Case (ii). There are no prescribed entries in the row $n$ and column $n$. By Theorem 2, there exists an $(n-1)$-square matrix $A^{\prime}=\left(a_{i j}^{\prime}\right)$, with distinct eigenvalues, satisfying $a_{i_{t} j_{t}}^{\prime}=a_{t}, t=1, \cdots, n-1$. Let

$$
S^{-1} A^{\prime} S=\operatorname{diag}\left(\beta_{1}, \cdots, \beta_{n-1}\right),
$$

where the $\beta_{i}$ are distinct. Set $a_{n}=\lambda_{1}+\cdots+\lambda_{n}-\beta_{1}-\cdots-\beta_{n-1}$ and consider the matrix

$$
M=\left[\begin{array}{ccccc:c}
\beta_{1} & & & & p_{1} \\
& & \cdot & & 0 & \vdots \\
0 & & \cdot & & \vdots \\
& & & \beta_{n-1} & p_{n-1} \\
\hdashline 1 & \cdot & \cdot & \cdot & 1 & a_{n}
\end{array}\right] .
$$

We use Mirsky's method [3, Proof of Theorem 1] and choose

where

$$
p_{j}=-f\left(\beta_{j}\right) / g^{\prime}\left(\beta_{j}\right), \quad j=1, \cdots, n-1,
$$

$$
f(x)=\prod_{i=1}^{n}\left(x-\lambda_{i}\right), \quad g(x)=\prod_{i=1}^{n-1}\left(x-\beta_{i}\right) .
$$

With this choice, the eigenvalues of $M$ are $\lambda_{1}, \cdots, \lambda_{n}$ [3]. The matrix

$$
A=(S+1) M\left(S^{-1}+1\right)=\left[\begin{array}{c:c}
A^{\prime} & S p \\
\hdashline e S^{-1} & a_{n}
\end{array}\right] \text {, }
$$

where $p=\left(p_{1}, \cdots, p_{n-1}\right)^{T}$ and $e=(1, \cdots, 1)$, has eigenvalues $\lambda_{1}, \cdots, \lambda_{n}$ and the prescribed entries in the prescribed positions.

It is clear that the number $n-1$ of prescribed entries cannot be increased in general. For example, if all the entries in the main diagonal are prescribed, then the eigenvalues cannot be arbitrarily prescribed. This is also the case if all the entries in a row (or in a column) are prescribed to be equal to zero. 


\section{REFERENCES}

1. H. K. Farahat and W. Ledermann, Matrices with prescribed characteristic polynomials, Proc. Edinburgh Math. Soc. 11 (1958/59), 143-146. MR 21 \#6382.

2. M. Marcus and H. Minc, $A$ survey of matrix theory and matrix inequalities, Allyn and Bacon, Boston, Mass., 1964. MR 29 \#112.

3. L. Mirsky, Matrices with prescribed characteristic roots and diagonal elements, J. London Math. Soc. 33 (1958), 14-21. MR 19, 1034.

Department of Mathematics, Technion-Israel Institute of Technology, HAIFA, ISRAEL

Department of Mathematics, University of California, Santa Barbara, CALIFORNIA 93106 